\title{
STUDIES ON THE MECHANISM OF ACTION OF AMANTADINE
}

\author{
F. BROWN \& P.H. REDFERN \\ School of Pharmacy and Pharmacology, University of Bath, Claverton Down, Bath BA2 7AY
}

1 The effect of amantadine hydrochloride on various aspects of catecholamine metabolism in the rat brain has been investigated.

2 Amantadine failed to have any significant effect on brain concentrations of dopamine or noradrenaline even when administered daily for 9 days.

3 Amantadine had no effect on the rate of decline of noradrenaline and dopamine concentrations after $\alpha$-methyl-p-tyrosine.

4 In vitro, amantadine inhibited dopamine uptake into synaptosomes only at high concentrations, and caused little release of dopamine from synaptosomes.

5 There is no evidence from these results to suggest that the anti-Parkinsonian effect of amantadine is related to an action on dopaminergic mechanisms.

\section{Introduction}

It is generally believed that the anti-Parkinsonian drug amantadine owes its effectiveness to a central action on dopaminergic mechanisms. However, there is a lack of concensus as to the exact mechanism of action of the drug. Direct stimulation of dopamine receptors (Papeschi, 1974), blockade of neuronal uptake (Fletcher \& Redfern, 1970; Heimans, Rand \& Fennessy, 1972; Baldessarini, Lipinski \& Chace, 1972), catecholamine release (Scatton, Cheramy, Besson \& Glowinski, 1970; Von Voigtlander \& Moore, 1971; Stromberg \& Svensson, 1971; Farnebo, Fuxe, Goldstein, Hamberger \& Ungerstedt, 1971) and increased synthesis (Scatton et al., 1970) have all been suggested to be responsible. We have investigated this problem by examining the effect of amantadine on brain concentrations of catecholamines, on the turnover of these amines in the brain, and on the uptake and release of dopamine from isolated synaptosome preparations. A preliminary report of these results was presented to the British Pharmaceutical Conference (Brown \& Redfern, 1974).

\section{Methods}

Estimation of noradrenaline and dopamine concentrations

Groups of 10 male Sprague-Dawley rats weighing 150 to $250 \mathrm{~g}$ received either $0.9 \% \mathrm{w} / \mathrm{v} \mathrm{NaCl}$ solution (saline) or amantadine intraperitoneally and were killed $0.5,1$ or $2 \mathrm{~h}$ after injection. The brains were quickly removed and homogenized in $8 \mathrm{ml}$ of $0.4 \mathrm{~N} \mathrm{HClO}_{4}$ containing $0.1 \% \quad \mathrm{Na}_{2} \mathrm{~S}_{2} \mathrm{O}_{3}$ and $0.05 \%$ disodium edetate. Homogenates were washed into ulta-centrifuge tubes with a further $1 \mathrm{ml}$ of $\mathrm{HClO}_{4}$ and, after centrifuging at $25,000 \mathrm{~g}$ for $15 \mathrm{~min}$, the clear supernatant was carefully decanted. The noradrenaline and dopamine concentrations in $6 \mathrm{ml}$ aliquots of the supernatants were assayed fluorimetrically after isolation on alumina (Shellenberger \& Gordon, 1971).

\section{Estimation of noradrenaline and dopamine turnover}

Groups of male Sprague-Dawley rats were injected intraperitoneally with $250 \mathrm{mg} / \mathrm{kg} \alpha$-methyl-p-tyrosine methyl ester hydrochloride (AMPT) and brain concentrations of noradrenaline and dopamine were estimated by the method described above in different groups of animals at intervals after injection. The rate of decline of dopamine and noradrenaline levels was calculated by log linear regression analysis of the results obtained during the $4 \mathrm{~h}$ after injection.

\section{Dopamine uptake into brain homogenates}

The method was based on that of Snyder \& Coyle (1969). Male Sprague-Dawley rats were killed by decapitation and the brains quickly removed and placed on ice. Whole brains or discrete brain areas were homogenized in $0.32 \mathrm{M}$ sucrose adjusted to pH 7.0 with sodium bicarbonate. using a teflon-in- 
glass homogenizer with $0.254 \mathrm{~mm}$ clearance, and the homogenates were centrifuged at $4^{\circ} \mathrm{C}$ at $1000 \mathrm{~g}$ for 10 minutes. Aliquots $(0.5 \mathrm{ml})$ of the synaptosome-rich supernatant were incubated with $4.5 \mathrm{ml}$ of modified Krebs solution for $5 \mathrm{~min}$ at $37^{\circ} \mathrm{C}$. After this period of equilibration, radioactively-labelled dopamine was added. The labelled dopamine bound by the synaptosomes was then recovered at various times using one of two methods: (i) $5.0 \mathrm{ml}$ of incubation medium at $0-4{ }^{\circ} \mathrm{C}$ was added to the incubation mixture and the tubes were centrifuged at $50,000 \mathrm{~g}$ for 20 minutes. The pellets were collected, washed and dispersed in $0.5 \mathrm{ml}$ of $0.5 \mathrm{M} \mathrm{NaOH}$. Aliquots of the digested pellets were taken for liquid scintillation counting in 'Unisolve' (Koch-Light). (ii) $0.5 \mathrm{ml}$ of the incubation mixture was added to $5.0 \mathrm{ml}$ of cold incubation medium and this mixture was quickly passed through a millipore membrane $(0.22 \mu \mathrm{m}$ pore size). After washing with a further $5.0 \mathrm{ml}$ of cold medium, the filter disc was dried and placed in a scintillation vial. The radioactive content of the dried membrane was measured by liquid scintillation counting after shaking with $0.5 \mathrm{ml}$ distilled water and adding $9.5 \mathrm{ml}$ 'Unisolve'.

\section{Dopamine release from synaptosomes}

The method was essentially the same as that used in the uptake experiments using millipore filters for rapid separation of the synaptosomes from the incubating medium. After a $5 \mathrm{~min}$ incubation the radioactive dopamine content of the synaptosomes reached a maximum. A significant decline in radioactive content

Table 1 The effect of amantadine on dopamine and noradrenaline concentrations in rat brain

\begin{tabular}{|c|c|c|c|c|c|c|c|}
\hline \multicolumn{8}{|c|}{ Dopamine $(n g / g \pm$ s.e. mean) } \\
\hline \multirow{2}{*}{$\begin{array}{l}\text { Time after } \\
\text { amantadine (h) }\end{array}$} & \multicolumn{6}{|c|}{ Dose of amantadine (mg/kg i.p.) } & No. of rats \\
\hline & 0 & 10 & 40 & 80 & 160 & $94 \mathrm{mg} / \mathrm{kg}$ per day & \\
\hline 0.5 & $787 \mp 23$ • & & & $791 \mp 12$ & & & 10,10 \\
\hline 1.0 & - $835 \mp 21$ & $806 \mp 21$ & & & & & 10,10 \\
\hline & $907 \mp 32$ & & $932 \mp 15$ & & & & 10,10 \\
\hline & $811 \mp 17$ & & & $790 \mp 16$ & & & 10,10 \\
\hline & $866 \mp 24$ & & & $788 \mp 18^{*}$ & & & 10,10 \\
\hline & $954 \mp 43$ & & & & $958 \mp 49$ & & 8,7 \\
\hline 2.0 & $\begin{array}{l}893 \mp 43 \\
776 \mp 22\end{array}$ & $979 \mp 29$ & & & & & 6,6 \\
\hline 3.0 & $\begin{array}{l}776 \mp 22 \\
712 \mp 36\end{array}$ & & $714 \mp 24$ & $\begin{array}{l}781 \mp 28 \\
739 \mp 42\end{array}$ & & & $\begin{array}{c}9,9 \\
9,8,8\end{array}$ \\
\hline $\begin{array}{l}\text { Chronic } \\
\text { treatment }\end{array}$ & $838 \mp 12$ & & & & & $774 \mp 30$ & 10,10 \\
\hline
\end{tabular}

Time after

amantadine (h)

0

$443 \mp 22$

0.5

1.0

$412 \mp 18$

$533 \mp 23$

$408 \mp 13$

$432 \mp 13$

$20 \quad 416 \mp 8$

$441 \mp 21$

3.0

$422 \mp 31$

Chronic

treatment

$421 \mp 10$
Noradrenaline (ng/g ₹s.e. mean)

Dose of amantadine (mg/kg i.p.)

No. of rats

per group

$40 \quad 80 \quad 160 \quad 94 \mathrm{mg} / \mathrm{kg}$ per day

$$
476 \mp 23
$$

10,10

10,10

10,10

10,10

10,10

8,7

6,6

9,9

$9,8,8$

$405 \mp 25352 \mp 32$

$$
402 \mp 19
$$

$430 \mp 16$
10,10 


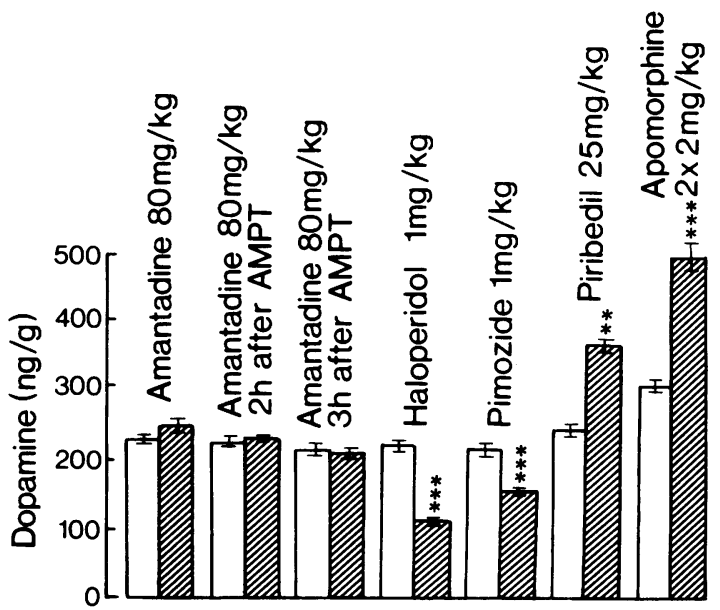

Figure 1 Dopamine concentrations in rat brain $4 \mathrm{~h}$ after $\alpha$-methyl-p-tyrosine (AMPT, $250 \mathrm{mg} / \mathrm{kg}$ i.p.). Open columns=AMPT alone; hatched columns =AMPT + drug. Vertical bars show s.e. means. Drugs were generally administered at the same time as AMPT. Apomorphine was given in two doses, one with the AMPT and one $2 \mathrm{~h}$ later.

Differences from corresponding controls: $* * P<0.01$; ***P$<0.001$

following the addition of drugs to the incubation mixture at this point was taken to indicate release of previously-bound radioactive dopamine from the synaptosomes.

\section{Drugs}

The following drugs were donated: amantadine hydrochloride (Geigy Pharmaceuticals Ltd.), rimantadine hydrochloride (E.T. Du Pont de Nemours and Co. Ltd.), piribedil methane sulphonate (Servier Laboratories Ltd.), apomorphine hydrochloride (Macfarlan Smith Ltd.), haloperidol (G.D. Searle and Co. Ltd.) and pimozide (Janssen Pharmaceuticals). (+)-Amphetamine sulphate and $\alpha$-methyl-p-tyrosine methylester hydrochloride were obtained from Sigma London Chemical Co. Ltd.

\section{Results}

Table 1 shows the concentrations of noradrenaline and dopamine in the rat brain after various doses of amantadine. It can be seen that in only one experiment was there any significant change in amine levels. After $80 \mathrm{mg} / \mathrm{kg}$ amantadine, dopamine concentrations were decreased while noradrenaline concentrations were increased. Both these changes were just significant at the $95 \%$ level, and were not seen when the experiment

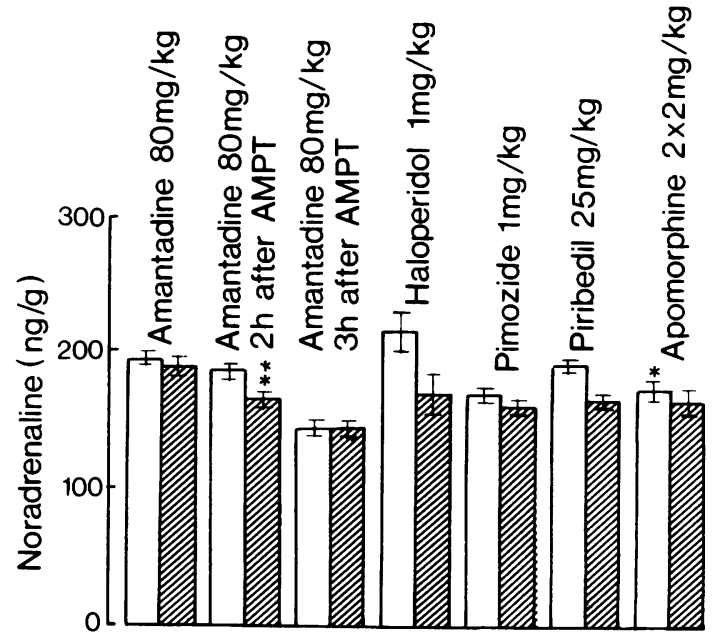

Figure 2 Noradrenaline concentrations in rat brain $4 \mathrm{~h}$ after $\alpha$-methyl-p-tyrosine (AMPT $250 \mathrm{mg} / \mathrm{kg}$ i.p.). Open columns=AMPT alone; hatched columns $=$ AMPT + drug. Vertical bars show s.e. means. The times of drug administration were the same as in Figure 1.

Differences from corresponding controls: $* P<0.05$; ${ }^{* *} P<0.01 ;{ }^{* * *} P<0.001$.

was repeated. Even when rats were treated for 9 days with amantadine (administered in the drinking water in an approximate daily dose of $94 \mathrm{mg} / \mathrm{kg}$ ) dopamine and noradrenaline levels were not significantly altered.

Figures 1 and 2 respectively show the effect of drugs on the rate of decline of dopamine and noradrenaline concentrations after AMPT. It can be seen that drugs which are believed to exert a direct action on dopamine receptors had a significant effect on dopamine turnover. For instance the dopamine antagonist pimozide, $1 \mathrm{mg} / \mathrm{kg}$, injected $30 \mathrm{~min}$ before AMPT significantly increased the rate of decline of dopamine concentrations $(P<0.001)$, while there was no change in noradrenaline concentrations. Haloperidol, $1 \mathrm{mg} / \mathrm{kg}$, had a similar effect. Conversely when dopamine receptor stimulants such as piribedil $25 \mathrm{mg} / \mathrm{kg}$ and apomorphine $2 \times 2 \mathrm{mg} / \mathrm{kg}$ were administered, the consequent inhibition of dopamine release produced an equally significant reduction in the rate of decline in dopamine concentration.

As can be seen from Figure 1, amantadine $(80 \mathrm{mg} / \mathrm{kg}$ i.p.) produced no measurable change in dopamine turnover whether administered simultaneously with the AMPT or $2 \mathrm{~h}$ or $3 \mathrm{~h}$ afterwards. A slight but statistically significant $(P<0.01)$ fall in noradrenaline levels was observed when amantadine $(80 \mathrm{mg} / \mathrm{kg})$ was administered $2 \mathrm{~h}$ after AMPT, but not when the same dose was given either at the same time as the AMPT or $3 \mathrm{~h}$ afterwards (Figure 2). 

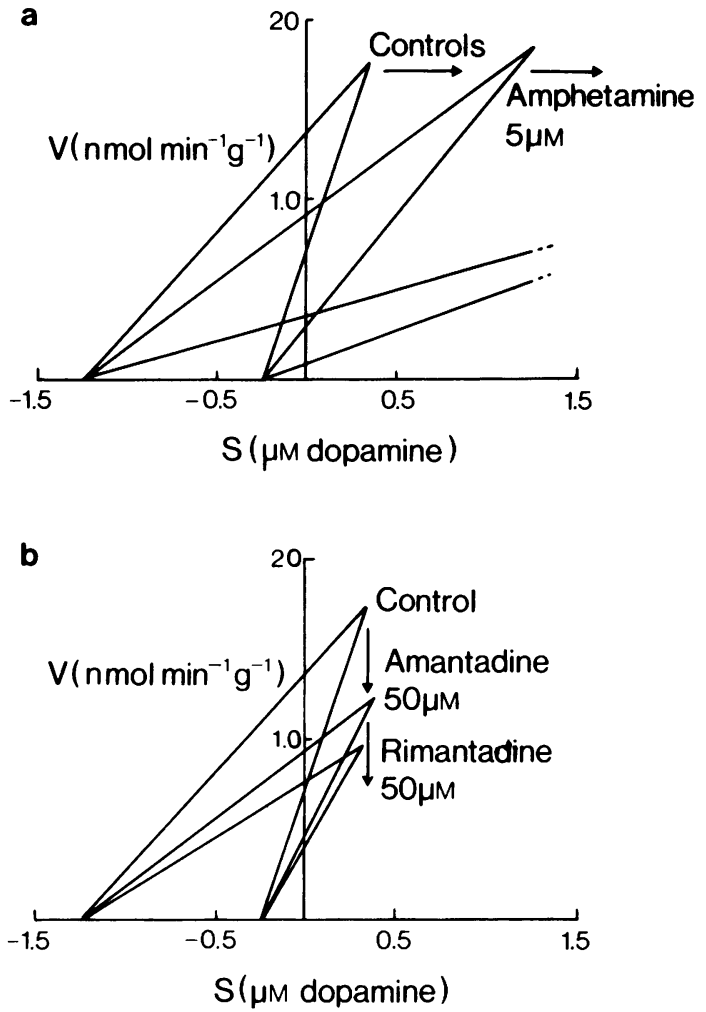

Figure 3 Direct linear plots (Eisenthal \& CornishBowden, 1974) of dopamine uptake into synaptosomes prepared from rat corpus striatum: (a) competitive inhibition produced by $(+)-$ amphetamine; (b) non-competitive inhibition produced by amantadine. Each line is the mean of 3 separate determinations at one substrate concentration.

\section{Effects of amantadine in vitro}

The effect of amantadine on dopamine uptake into synaptosomes, displayed according to the method of Eisentahl \& Cornish-Bowden (1974) is compared to that of (+)-amphetamine and of rimatadine in Figure 3. Both amantadine and rimantadine, a close analogue of amantadine lacking clinically useful antiParkinsonian properties, are shown to be relatively weak noncompetitive inhibitors of dopamine uptake, with $K i$ values of $125 \mu \mathrm{M}$ and $69.9 \mu \mathrm{M}$ respectively. Amphetamine was a relatively potent competitive inhibitor, with a $K i$ of $1.94 \mu \mathrm{M}$.

In spite of considerable differences in control figures for dopamine uptake in different brain regions, especially between those of the striatum and other areas, amantadine caused a similar inhibitory effect, relative to controls, in all areas. Amantadine also failed to show any selective effect against dopamine

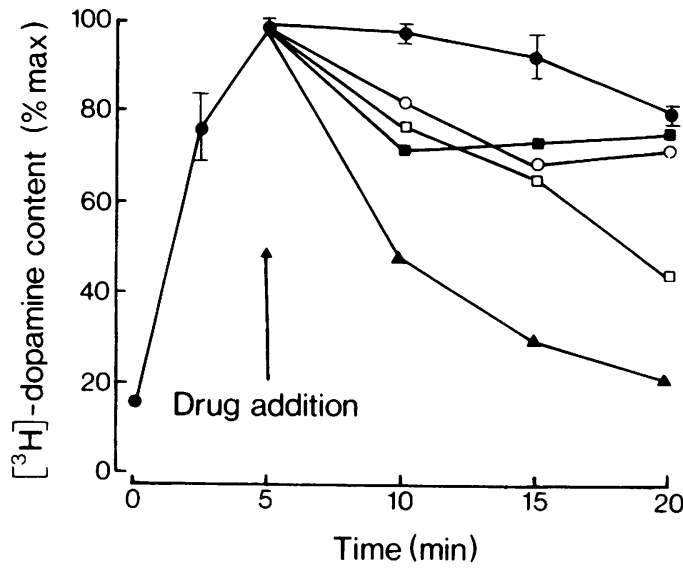

Figure 4 Effect of drugs on release of radioactive dopamine from synaptosomes prepared from rat corpus striatum: (O) control; ( $\square$ ) amantadine $100 \mu \mathrm{M}$; (O) piribedil $100 \mu \mathrm{M}$; (ㅁ) ouabain $100 \mu \mathrm{M}$; (A) tyramine $10 \mu \mathrm{M}$.

uptake compared to uptake of either noradrenaline or 5-hydroxytryptamine. Finally, pretreatment of rats with amantadine $(50 \mathrm{mg} / \mathrm{kg}$ i.p. $) 1 \mathrm{~h}$ before preparation of homogenates did not result in any significant inhibition of dopamine uptake as compared to saline pretreated controls.

$\left[{ }^{3} \mathrm{H}\right]$-dopamine uptake into striatal homogenates reached a maximum after about 5 min incubation. Thereafter there was a gradual decline. Figure 4 shows the effect of adding various drugs to the incubation mixture after $5 \mathrm{~min}$ incubation. It can be seen that neither amantadine $100 \mu \mathrm{M}$ nor piribedil $100 \mu \mathrm{M}$ produced any marked decline in $\left[{ }^{3} \mathrm{H}\right]$-dopamine content, when compared to the effect of tyramine $10 \mu \mathrm{M}$. Indeed, the decline in $\left[{ }^{3} \mathrm{H}\right]$-dopamine content produced by ouabain $100 \mu \mathrm{M}$ was more marked than that due to amantadine or piribedil.

\section{Discussion}

Despite the numerous reports of the clinical efficacy of amantadine in Parkinson's disease, the mode of action of the drug remains unclear. Since the drug has minimal antimuscarinic properties (Vernier, Harman, Stump, Lynes, Marvel \& Smith, 1969), some action on dopaminergic mechanisms seems most likely to account for its clinical action, yet no consistent evidence as to the exact nature of this action exists. For instance, various workers have used a variety of methods to estimate brain amines in the rat after amantadine treatment. Baldessarini et al. (1972) reported a fall in dopamine levels, while Stromberg \& Svensson (1971), Abuzzahab (1971), Bak, Hassler, 
Kim \& Kataoka (1972), Maj, Sowinska \& Baran (1972) and Papeschi (1974) all reported unchanged dopamine levels. Similarly while Baldessarini et al. (1972) and Stromberg \& Svensson (1971) found decreased concentrations of noradrenaline after amantadine, Abuzzahab (1971), Maj et al. (1972) and Papeschi (1974) found no change. In our experiments, despite the fact that both the time scale and dose range studied included those at which maximal behavioural effects occur (Stromberg \& Svensson, 1971; Maj et al., 1972; Davies, Jackson \& Redfern, 1973) no significant changes in noradrenaline or dopamine concentrations were obtained even after chronic treatment.

In the experiments with AMPT, it is evident that drugs which stimulate dopamine receptors, such as apomorphine (Ernst, 1967) or piribedil (Costall \& Naylor, 1973) caused slowing of dopamine turnover, while receptor blocking drugs like haloperidol and pimozide (Janssen, 1967, Anden, Butcher, Corrodi, Fuxe \& Ungerstedt, 1970) caused an acceleration of turnover. The simplest interpretation of these results is feedback inhibition or activation of dopaminergic neurones, presumably in an attempt to maintain homeostasis, because the changes in turnover are dependent on nerve impulse activity (Anden, Fuxe \& Hokfelt, 1966).

The failure of amantadine to modify dopamine turnover under these circumstances, when compared to the effectiveness of the dopamine receptor stimulants and blockers indicates that amantadine caused no significant activation of postsynaptic dopamine receptors. Such an action has been proposed by Papeschi (1974) who found a higher level of dopamine in rats given amantadine $1 \mathrm{~h}$ before AMPT than in animals given only AMPT. However, this effect was only observed at an early stage $(1 \mathrm{~h})$ after AMPT administration, when the response is complicated by non-linear decay (Glowinski, 1972).

There are similarly conflicting data from behavioural experiments on the contribution of pre- or postsynaptic events to the action of amantadine. BuusLassen $(1971$; 1973) found that locomotor activity due to amantadine was unaffected by pretreatment with AMPT. These results appear to support a postsynaptic action for amantadine, as do similar results of Maj et al. (1972) and Thornburg \& Moore (1972). In contrast, results on turning behaviour from unilaterally striectomised animals demonstrate that in this experimental model an indirect or presynaptic action is responsible for the effects of amantadine (Farnebo et al., 1971; Von Voigtlander \& Moore, 1973). Since rotational behaviour is more specific for striatal dopaminergic function while both dopamine and noradrenaline may contribute to locomotor responses, we believe the behavioural results are consistent with the results reported here, suggesting a presynaptic action for amantadine. However, the results of our in vitro experiments do not provide any clear indication of the exact nature of such an action.

Amantadine was not outstanding as an inhibitor of dopamine uptake into synaptosomes in vitro, producing significant inhibition only at high doses ( $50 \%$ inhibition at $100 \mu \mathrm{M})$. This finding is supported by results of other workers (Baldessarini et al., 1972; Heikkila \& Cohen, 1972; Herblin, 1972) although Heimans et al. (1972) reported that amantadine would cause $50 \%$ inhibition of dopamine uptake into striatal homogenates at a concentration as low as $3.6 \mu \mathrm{M}$.

Direct linear plots of the relationship between the rate of dopamine uptake and the initial dopamine concentration, calculated by the method of Eisenthal \& Cornish-Bowden (1974) in which substrate concentration is plotted on the abscissa and the velocity on the ordinate scale, showed that in the presence of amantadine, Vmax is displaced vertically downwards, indicating a non-competitive inhibitory effect. In contrast, in the presence of amphetamine the apparent $K_{m}$ was displaced to the right, indicating competitive inhibition. The fact that amphetamine, a drug generally considered to be a dopamine releasing agent, should apparently be an effective inhibitor of uptake could indicate that the system used is non-specific, i.e. a sudden release of dopamine could give the same result in terms of relative distribution of radioactivity as inhibition of uptake. However, Raiteri, Levi \& Federico (1974) showed that, using a superfusion technique, amphetamine was indeed a powerful inhibitor of noradrenaline uptake and had a minimal effect on release. In the in vitro system used in these experiments amphetamine, haloperidol, benapryzine and the amantadine analogue, rimantadine, all inhibited dopamine uptake more effectively than did amantadine. This relative ineffectiveness of amantadine, together with the fact that the drug was no more effective in blocking dopamine uptake than in blocking noradrenaline or 5-hydroxytryptamine uptake suggests that this mechanism is unlikely to account for the anti-Parkinsonian action of amantadine.

A dopamine releasing property for amantadine has been claimed by many workers (Stromberg, Svensson \& Waldeck, 1970; Stromberg \& Svensson, 1971; Farnebo et al., 1971) while others have found it to be a less effective releasing agent than uptake blocking agent (Heikkila \& Cohen, 1972; Heimans et al., 1972). As has already been mentioned, it is difficult in an in vitro system such as we have used to distinguish between uptake blockade and amine release. Nevertheless comparison of amantadine with other drugs known either to cause dopamine release or to inhibit dopamine uptake provides a reasonable basis for conjecture. On this basis, since a significant decline in dopamine concentration was caused by tyramine, whereas the response to amantadine was less marked even than that to ouabain, it is concluded 
that amantadine has negligible ability to release dopamine from nerve endings, at least in vitro.

The results reported, in common with those of many other workers, appear to show that if amantadine does have an effect on central dopaminergic mechanisms, the effect is a slight one and is certainly not likely to be evident at clinically effective dose levels. Two suggestions can be made from these results. First it is possible that the antiParkinsonian activity of amantadine is due to mechanisms unrelated to brain dopamine. Certainly, Bak et al. (1972) have shown an increase in striatal acetylcholine and $\gamma$-aminobutyric acid concentrations in rat, although high doses $(100 \mathrm{mg} / \mathrm{kg})$ were used, while in man, amantadine has been shown to cause an increase in the excretion of 1,4-methylhistamine (Jones, Turnbull, Lenman \& Robertson, 1972). The second possibility is that the effect of amantadine on brain dopaminergic mechanisms only becomes evident under the conditions obtaining in a Parkinsonian patient. For instance it has been reported that the increase in excretion of dopamine metabolites

\section{References}

ABUZZAHAB, F.S. (1971). Effects of haloperidol on rat brain catecholamines and behaviour. Fedn Proc., 381.

ANDEN, N.E., BUTCHER, S.G., CORRODI, H., FUXE, K \& UNGERSTEDT, V. (1970). Receptor activity and turnover of dopamine and noradrenaline after neuroleptics. Eur. J. Pharmac., 11, 303-314.

ANDEN, N.E., FUXE, K. \& HOKFELT, T. (1966). The importance of the nervous impulse flow for the depletion of the monoamines from central neurones by some drugs. J. Pharm. Pharmac., 18, 630-632.

BAK, I.J., HASSLER, R., KIM, J.S. \& KATOAKA, K. (1972). Amantadine actions on acetylcholine and GABA in striatum and substantia nigra of rat in relation to behavioural changes. J. Neurol. Trans., 33, 45-61.

BALDESSARINI, R.J., LIPINSKI, B.F. \& CHACE, K.V. (1972). Effects of amantadine $\mathrm{HCl}$ on catecholamine metabolism in the brain of the rat. Biochem. Pharmac., 21, 77-87.

BROWN, F. \& REDFERN, P.H. (1974). The effect of amantadine on the turnover of catecholamines in the rat brain. J. Pharm. Pharmac., 26, 81-82P.

BUUS-LASSEN, J. (1971). Behavioural effect of amantadine in rats after inhibition of monoamine synthesis, storage and receptor interaction. Acta pharmac. tox., 29 Suppl. 4, 30.

BUUS-LASSEN, J. (1973). The effect of amantadine and (+)amphetamine on motility in rats after inhibition of monoamine synthesis and storage. Psychopharmacologia, 29, 55-64.

COSTALL, B. \& NAYLOR, R.J. (1973). The site and mode of action of ET495 for the mediation of stereotyped behaviour in the rat. Naunyn-Schmeidebergs Arch. Pharmak., 278, 117-133.

DAVIES, J.A. \& REDFERN. P.H. (1974). The interaction of produced by amantadine in Parkinsonian patients is not reproduced in non-Parkinsonian volunteers (Jones et al., 1972), indicating that in man, the effect of amantadine may only be demonstrable in the presence of the underlying biochemical abnormalities associated with Parkinson's disease. Secondly, in some behavioural tests, amantadine can be shown to exert a powerful and apparently specific dopaminergic action (Davies et al., 1973; Davies \& Redfern, 1974). It is clear that the experimental models used here utilize different facets of normal dopaminergic transmission, and that in order to establish the mode of action of amantadine an experimental model incorporating the essential biochemical lesions of Parkinson's disease may be required.

This work was supported by a CASE studentship; the joint sponsorship of the SRC and Geigy Pharmaceuticals Ltd. is gratefully acknowledged. F.B. is a Parkinson's Disease Society Post Doctoral Research Fellow. We wish to thank the Drug Companies for generous donations of the drugs mentioned in the Methods Section. dopamine antagonists and amantadine $\mathrm{HCl}$ on the acquisition of the conditioned avoidance response in rats. Neuropharmac., 13, 941-948.

DAVIES, J.A., JACKSON, B. \& REDFERN, P.H. (1973). Effect of anti-Parkinsonian drugs on haloperidol-induced inhibition of the conditioned avoidance response in rats. Neuropharmac., 12, 735-740.

EISENTHAL, R. \& CORNISH-BOWDEN, A. (1974). The direct linear plot: a new graphical procedure for estimating enzyme kinetic parameters. Biochem. J., 139, $715-720$.

ERNST, A.M. (1967). Mode of action of apomorphine and dexamphetamine on gnawing compulsion in rats. Psychopharmacologia, 10, 316-323.

FARNEBO, L., FUXE, K., GOLDSTEIN, M., HAMBERGER, B. \& UNGERSTEDT, U. (1971). Dopamine and noradrenaline releasing action of amantadine in the central and peripheral nervous system: a possible mode of action in Parkinson's disease. Eur. J. Pharmac., 16, 27-38.

FLETCHER, E.A. \& REDFERN, P.H. (1970). The effect of amantadine on the uptake of dopamine and noradrenaline by rat brain homogenates. J. Pharm. Pharmac., 22, 957-959.

GLOWINSKI, J. (1972). Some new facts about synthesis, storage and release processes of monoamines in the central nervous system. In Perspectives in Neuropharmacology, ed. Snyder, S.H., pp. 349-404. London: Oxford University Press.

HEIKKILA, R.E. \& COHEN, G. (1972). Evaluation of amantadine as a releasing agent or uptake blocker for ${ }^{3} \mathrm{H}$-dopamine in rat brain slices. Eur. J. Pharmac., 20, $156-160$.

HEIMANS, R.L.H., RAND, M.J. \& FENNESSY, M.R. (1972). 
Effects of amantadine on uptake and release of dopamine by a particulate fraction of rat basal ganglion. J. Pharm. Pharmac., 24, 875-879.

HERBLIN, W.F. (1972). Amantadine and catecholamine uptake. Biochem. Pharmac., 21, 1993-1995.

JANSSEN, P.A.J. (1967). The pharmacology of haloperidol. Int. J. Neuropsychiat., 3 Suppl. 1, 510-518.

JONES, D.G., TURNBULL, M.J., LENMAN, J.A.R. \& ROBERTSON, M.A.H. (1972). Effect of amantadine on the urinary excretion of some monoamines and metabolites in normal and Parkinsonian subjects. $J$. Neurol. Sci., 17, 245-253.

MAJ, J., SOWINSKA, H. \& BARAN, L. (1972). The effect of amantadine on motor activity and catalepsy in rats. Psychopharmacologia, 24, 296-307.

PAPESCHI, R. (1974). Amanatadine may stimulate dopamine and noradrenaline receptors. Neuropharmac., 13, 77-84.

RAITERI, M., LEVI, G. \& FEDERICO, R. (1974). damphetamine and the release of ${ }^{3} \mathrm{H}$-norepinephrine from synaptosomes. Eur.J. Pharmac., 28, 237-240.

SCATTON, B., CHERAMY, A., BESSON, M.J. \& GLOWINSKI, J. (1970). Increased synthesis and release of dopamine in the striatum of the rat after amantadine treatment. Eur. J. Pharmac., 13, 131-133.

SHELLENBERGER, M.K. \& GORDON, J.H. (1971). A rapid simplified procedure for simultaneous assay of norepirephrine, dopamine and 5-hydroxytryptamine from discrete brain areas. Analyt. Biochem., 39, 356-372.

SNYDER, S.H. \& COYLE, J.T. (1969). Regional differences in $\mathrm{H}^{3}$-norepirephrine and $\mathrm{H}^{3}$-dopamine uptake into rat brain homogenates. J. Pharmac. exp. Ther., 165, 78-86.

STRÖMBERG, U. \& SVENSSON, T.H. (1971). Further studies on the mode of action on amantadine. Acta pharmac. tox., 30, 161-171.

STRÖMBERG, U., SVENSSON, T.H. \& WALDECK, B. (1970). On the mode of action of amantadine. J. Pharm. Pharmac., 22, 959-962.

THORNBURG, J.E. \& MOORE, K.E. (1972). A comparison of the locomotor stimulant properties of amantadine and 1and d- amphetamine in mice. Neuropharmac., 11, 675-682.

VERNIER, V.G., HARMON. J.B., STUMP, J.M., LYNES, T.E., MARVEL, J.P. \& SMITH, D.H. (1969). The toxicologic and pharmacologic properties of amantadine hydrochloride. Toxicol. Appl. Pharmac., 15, 642-665.

VON VOIGTLANDER, P.F. \& MOORE, K.E. (1971). Dopamine release from the brain in vivo by amantadine. Science, 174, 408-410.

(Received March 29, 1976. Revised June 18, 1976.) 